\title{
An exploratory examination of mindfulness, self-compassion, and mindful eating in relation to motivations to eat palatable foods and BMI
}

\section{BACKGROUND}

Preliminary findings suggest that mindfulness and selfcompassion training are associated with enhanced weight regulation. However, the associations between these traits and body mass index (BMI) are mixed.

\section{PARTICIPANTS AND PROCEDURE}

In a cross-sectional investigation, university students ( $n=183$ ) were asked to fill in questionnaires on mindfulness, self-compassion, mindful eating, and motivations to eat palatable foods.

\section{RESULTS}

The results suggest that mindfulness, self-compassion and mindful eating related negatively to motivations to eat palatable foods. Mindful eating displayed the most significant relationship. Further investigations showed that some subscales of self-compassion, mindfulness and mindful eating related to motivations to eat palatable foods and BMI more significantly. The ability to draw more and better conclusions by investigating the relationship of sub- scales to health behaviors and outcomes, especially with self-compassion, has been noted in previous rationales and theories. The notable relationships were (a) the enhancement subscale of the motivations to eat palatable foods, which appeared to relate positively to self-judgment, isolation, and over-identification, while (b) the coping subscale related to all subscales within the self-compassion scale.

\section{CONCLUSIONS}

The current findings support different lines of research that suggest that mindful eating, mindfulness and/or self-compassion support weight regulation. Mindfulness practices could potentially add the right motives to eat palatable foods (such as being motivated to eat when people are hungry), and potentially eat less of the foods that lead to weight dysregulation. The findings are discussed and suggested paths for further research are recommended.

\section{KEY WORDS}

mindfulness; self-compassion; obesity; palatable foods; coping 


\section{BACKGROUND}

Recent research in the field of mindfulness and self-compassion suggests that there is some merit in developing those traits by utilizing the corresponding practices for obesity prevention and weight regulation. While the psychological benefits, as well as the aligned health behaviors, are well documented (e.g., Neff, 2011; Schoenefeld \& Webb, 2013), and practices and interventions show promising results (e.g., Mantzios \& Wilson, 2014), associations with body mass index (BMI) have been mixed and inconclusive. This inconsistency in findings creates uncertainty as to whether those traits are useful for weight regulation, when considered separately from disordered eating and active interventions.

Based on a recent review exploring the relationship between self-compassion and obesity (see Mantzios \& Egan, 2017), it was suggested that reporting findings from the subscales of those traits might offer further insights. Another review suggested investigating mindful eating more specifically, to identify more relevant and useful associations in obesity research (Mantzios \& Wilson, 2015a). The overall aim of this research is to clarify the relationship of mindfulness, mindful eating and self-compassion to BMI through the use of a recent scale that assesses the motivations behind eating palatable foods. The relationship of mindfulness, mindful eating and self-compassion with motivations to eat palatable foods has not been explored, and might offer supplementary understanding of their association with BMI. Two methods of investigation have been used in this research. First, BMI is explored with the overall scores of mindfulness, mindful eating, and self-compassion, while considering the association with motivations to eat palatable foods. Second, BMI is explored in relation to the subscales of the mindfulness, mindful eating, self-compassion and motivations to eat palatable foods scales.

\section{MINDFULNESS, EATING, AND OBESITY}

The practice of mindfulness is described as an awareness that emerges through purposefully paying attention in the present moment, non-judgmentally (Kabat-Zinn, 1990). Practice usually entails attentional training which is executed through mindfulness meditation. Mindfulness meditation involves actively observing the present moment by attending to the breath, moment to moment, and without adding any meaning to the feelings and thoughts that emerge. This process assists people who observe the constant flow of information unfolding in the present moment and to systematically develop an ability to accept (instead of judge) the experiences that are encountered. Working with mindfulness meditation practices has been shown to lead to other multi-layered indirect benefits, such as compassion, self-compassion and equanimity, which are parts of advanced mindfulness practice (Kabat-Zinn, 2006; Grossman \& Van Dam, 2011).

Mindfulness had been associated with health benefits, self-regulation and behavior modification. For example, a negative relationship was found between mindfulness and BMI in a recent population study with French adults $(N=63,628$ - see Camilleri, Méjean, Bellisle, Hercberg, \& Péneau, 2015). However, other studies did not make similar observations in relation to BMI and weight loss (Fuller et al., 2016; Hilbert et al., 2015). The question is whether French adults interpret the items in mindfulness scales differently (considering the 'French Paradox'; see Ferrières, 2004), or whether some other cultural elements are responsible for non-significant associations in other Western countries. While the literature is mixed in regards to the cross-sectional associations, it has been found that people who eat more mindfully, or participate in mindfulness meditation programs, improve the way they eat and successfully eat less. There are several studies with promising results that demonstrate the effectiveness of mindfulness-based interventions (Alberts, Mulkens, Smeets, \& Thewissen, 2010; Daubenmier et al., 2012; Kristeller \& Hallett, 1999; Kristeller, Wolever, \& Sheets, 2014; Mantzios \& Giannou, 2014; Mantzios \& Wilson, 2014; Mantzios et al., 2015). However, the relationship between BMI and mindfulness remains unclear, which may be attributable to several reasons including small sample sizes. Mantzios and Wilson (2014) suggested that the combination of mindfulness and self-compassion might be more beneficial than mindfulness alone, and this was subsequently evidenced in other studies (Mantzios \& Wilson, 2015b).

\section{SELF-COMPASSION, EATING AND OBESITY}

Self-compassion has been defined as a mindful awareness of oneself, which involves treating oneself kindly and understanding oneself during difficult and challenging times by realizing that such experiences are common among all humans (Neff, 2003a). Neff (2003a, 2003b) described how self-compassion consists of three interrelated units: self-kindness (vs. self-judgment), common humanity (vs. isolation), and mindfulness (vs. over-identification). These components combined create the construct of self-compassion (see Neff, 2003b; see also Neff, 2011).

Empirical studies exploring self-compassionate-based interventions have focused on weight management. For example, a recent study found that the trait of self-compassion negatively predicted weight gain in civilians who entered a stressful 
military environment (Mantzios, Wilson, Linnell, \& Morris, 2015). Another study found that participants lost more weight when they were assigned to a mindful self-compassionate program compared to a control group (Mantzios \& Wilson, 2015a). However, participants in these studies were not typical populations that are usually investigated in weight loss and weight regulation trials. They exercised regularly and had a controlled diet, while the confined environment created a particular experimental setting which resulted in restricting generalizability of these findings; therefore, further research is needed that can be more readily extrapolated. Self-compassion remains a trait that requires further research, merely because it is uncertain whether self-compassion leads to healthy choices around food, despite the weight loss that has been observed. Overall, the findings on self-compassion and weight regulation are inconsistent; specifically, self-compassion does not relate to BMI. While some preliminary findings were significant and promising in intervention studies, follow-up research is warranted to establish how self-compassion relates to BMI.

\section{MINDFUL EATING AND OBESITY}

The combination of mindfulness and eating has created a new drive for researchers who are specifically interested in investigating eating in terms of the principles of mindfulness: namely, mindful eating. Mantzios and Wilson (2015a) suggested in a recent review that investigations need to be more explicit and specific to eating. Mindful eating is the application of mindfulness fundamentals on food-related experiences; that is, purposeful attention to the present moment with a non-judgmental or accepting attitude. Mindful eating has been related to healthier eating (Jordan, Wang, Donatoni, \& Meier, 2015), and has been suggested to reduce glucose levels and assist weight loss through mindfulness-based interventions (Mason et al., 2013), but remains a construct that is not clearly related to lower BMI in cross-sectional research. Mindful eating (rather than mindfulness) is anticipated to relate more to being motivated to eat palatable foods, but has not yet been explored. Similarly, neither mindfulness nor self-compassion and the relationship with motives to eat palatable foods have been explored.

\section{MOTIVATIONS TO EAT PALATABLE FOODS AND OBESITY}

Pleasure-inducing foods are often fast foods, sweets and snacks that are of high calorific value which contribute to the weight gain and obesity observed in Western populations. While those foods may be eaten for reasons that relate to hunger (which is consistent with mindful eating practices), there are frequently other reasons for consumption. Knowing more about the motives or drives for eating these foods is key to understanding both the rising prevalence and poor efficacy in interventions for obesity. Recently, Burgess, Turan, Lokken, and Boggiano (2014) proposed a Palatable Eating Motives Scale (PEMS), which explores non-hunger driven motives such as coping, reward enhancement, social motives and conformity. While controlling for a number of factors including age, sex, ethnicity, binge-eating status and food addiction, the results indicated that coping was associated with higher BMI in a college and a weight-loss seeking sample (Boggiano et al., 2014; Burgess et al., 2014). Boggiano and colleagues (2014) also found that Coping, Reward Enhancement, and Conformity motives were associated with increased binge-eating severity. The PEMS was also recently used to predict a change in BMI over time in a longitudinal study, and an increase or decrease in Coping predicted future weight gain or loss, correspondingly (Boggiano et al., 2015).

Given the mixed results for relations between mindfulness, self-compassion and BMI, the association between BMI and motives to eat palatable foods, and the corresponding relation of the proposed personality constructs, we seek to offer more insights to explain the effectiveness of mindfulness-based interventions. Therefore, this research aimed primarily to explore the relationship between mindfulness, mindful eating, self-compassion and BMI, in relation to motives to eat palatable foods, and secondly, as suggested by previous literature, to investigate BMI and motives to eat palatable foods in relation to the subscales that constitute the traits of mindfulness, mindful eating and self-compassion. Because mindful eating is more behaviorally specific, we firstly hypothesized that mindful eating would relate more significantly (than mindfulness and self-compassion) to lower BMI and motivations to eat palatable foods. Second, and similar to the first hypothesis, mindful eating subscales should display a more significant negative relationship to BMI and motivations to eat palatable foods than mindfulness and self-compassion subscales.

\section{PARTICIPANTS AND PROCEDURE}

An online invitation to take part in a study investigating eating behaviors was forwarded to 226 undergraduate psychology students in their second year at university. One-hundred and eighty-three students were recruited who agreed to take part. Thirty-one participants were excluded from the sample, as their BMI was lower than 18, as it may have been an indication of the existence of an eating disorder; leav-
Motivations to eat palatable foods and mindfulness-based constructs 
Michail Mantzios, Helen Egan ing a sample of 152 undergraduate students. Participants $\left(M_{\text {age }}=24.40, S D=9.70, M_{\mathrm{BMI}}=24.70, S D=5.40\right.$, 134 females) were recruited on a voluntary basis and did not participate for any course credits or financial reward. Following the National Health Service consensus on ethnic background, this sample consisted of $51.90 \%$ British White, 32.30\% British Asian (14.80\% Pakistani, 14.80\% Indian, and 2.70\% Bangladeshi British), $6.00 \%$ Mixed, $2.70 \%$ Black British, and $4.40 \%$ Other ethnic background (i.e., 2.20\% Chinese and $2.20 \%$ Arab). Twelve percent of participants disclosed that they were currently smoking, and $52.00 \%$ indicated that they were currently exercising.

Participant information form. Participants' age, gender, ethnicity, socio-economic status, smoking and exercise status, including frequency, were recorded. Participants were also asked to report height and weight, by using the height recorded on their official identification (i.e., national identity card, passport, or driver's license) and to weigh themselves without any excess clothing and barefoot. BMI was calculated with the formula: weight in $\mathrm{kg} /$ height in $\mathrm{m}^{2}$. Questions regarding medication, health status, and eating disorders were also asked to serve for exclusion purposes.

Self-Compassion Scale (SCS; Neff, 2003). The scale calculates the qualities of the self-compassion construct. Responses range from 1 (almost never) to 5 (almost always). Sample items are "When I'm feeling down I tend to obsess and fixate on everything that's wrong" (i.e., over-identification) and "I try to be understanding and patient toward aspects of my personality I don't like" (i.e., self-kindness). It is a 26item scale (with overall scores ranging from 26 to $130)$ and it is composed of six subscales: self-kindness $(\alpha=.89)$, self-judgment $(\alpha=.84)$, common humanity $(\alpha=.89)$, isolation $(\alpha=.83)$, mindfulness $(\alpha=.84)$, and over-identification $(\alpha=.80)$. The present study produced an $\alpha$ of .95 for the original self-compassion scale, and the $\alpha$ for the subscales are represented within the parentheses in the previous sentence.

Five Facet Mindfulness Questionnaire - Short Form (FFMQ-SF; Bolhmeijer, Klooster, Fledderus, Veehof, $\&$ Baer, 2011). The FFMQ-SF is a 24-item questionnaire measuring five main characteristics of mindfulness. All items are scored on a 5-point Likert-type scale ranging from 1 (never or rarely true) to 5 (very often or always true). Sample items are "I watch my feelings without getting carried away by them" and "I find it difficult to stay focused on what's happening in the present moment", and higher scores indicate higher levels of mindfulness. The questionnaire is based on the original 39-item version (FFMQ; Baer, Smith, Hopkins, Krietemeyer, \& Toney, 2006). The five measured facets produced the following $\alpha$ values: observing $\alpha=.67$, describing $\alpha=.83$, acting with awareness $\alpha=.80$, non-judging $\alpha=.70$ and non-reactivity $\alpha=.75$. The present study produced an $\alpha$ of .78 for the overall score.
Mindful Eating Scale (MES; Hulbert-Williams, Nicholls, Joy, \& Hulbert-Williams, 2014). The MES is a 28 -item scale, with a Likert scale ranging from 1 (never) to 4 (usually). Higher scores represent higher mindful eating. Sample items are "When I feel anxious, I find myself eating" and "I stay aware of my food whilst I'm eating”. Five subscales were indicated by the developers of the scale following a factorial analysis: Acceptance $(\alpha=.88)$, Awareness $(\alpha=.81)$, Non-Reactivity $(\alpha=.80)$, Routine $(\alpha=.76)$, Distractibility $(\alpha=.88)$, and Unstructured $(\alpha=.75)$. The present study produced an overall $\alpha$ of .83 for the total score.

The Palatable Eating Motives Scale (PEMS; Boggiano et al., 2014). The PEMS is a 19-item Likert-like five-choice frequency response scale scored 1 (never) almost never) to 5 (always/almost always). The instructions ask how often tasty foods or drinks are consumed "for the following reasons", followed by the 19 reasons (items). The instructions provide examples of what is meant by "tasty foods" in categories that include various examples of sweets, salty snacks, fast food, fatty foods, and sugary drinks (Burgess et al., 2014). The PEMS evaluates 4 categories of motives: Coping, Reward Enhancement, Social, and Conformity. Coping motives include consuming these foods/drinks in an effort to deal with a negative state or situation (e.g., to forget about worries). Reward Enhancement motives relate to consuming these foods/drinks to enhance positive states or situations or for their inherently rewarding properties, e.g., "because it is fun". Social motives pertain to eating these foods/drinks to be more sociable or enhance enjoyment of gatherings, e.g., "to enjoy a party". Conformity motives pertain to eating these foods/drinks because of pressures by others to do so, e.g., "to fit in". Scores for each motive are calculated from the mean of the response values for items comprising each motive. Note that the first publication of the PEMS (Burgess et al., 2014) used the sum of response values rather than the mean but the mean is now the standard scoring method (Boggiano et al., 2014). A total PEMS score is obtained by summing the mean scores of each motive. In the present administration of the PEMS, individual motives had good internal reliability with Cronbach's $\alpha$ of .92, and an $\alpha$ of .90 for Coping, an $\alpha$ of .88 for Reward Enhancement, an $\alpha$ of .86 for Social, and an $\alpha$ of .86 for Conformity.

\section{PROCEDURE AND DESIGN}

Potential participants responded to an advertisement of various online invitations at a University in the West Midlands of the United Kingdom. Participants were able to click on a link, which directed them to a participant information form, a consent form, and 
was followed by the questionnaires and the demographic information page. Participants were aware that the research related to eating behaviors, but were blind to the mindfulness-based components of the questionnaire until they reached the debriefing page. At the end of the study participants were directed to a debriefing form, which allowed them to learn more around the current investigation, and gave the opportunity to participants to record an arbitrary number to withdraw at a later stage and retain anonymity. Ethical approval was granted by the Ethical Committee based within the university and care was taken that it strictly adhered to ethical guidelines set by the British Psychological Society.

The data were analyzed through inferential statistics and correlations, and the potential of mindfulness, mindful eating and self-compassion having a moderating/mediating role in the relationship between motives to eat palatable foods and BMI was explored.

\section{ETHICAL STANDARDS}

The study was approved by the Ethical Review Board of Birmingham City University, and was in accordance with the ethical standards of the institutional and/or national research committee, and with the 1964 Helsinki Declaration and its later amendments. Informed written consent was obtained prior to the experiment. This article does not contain any studies with animals.

\section{RESULTS}

Inter-correlations between mindfulness, mindful eating, self-compassion, motives to eat palatable foods and BMI are presented in Table 1. The findings suggest that there is a significant positive relationship between BMI and motivations to eat palatable foods, while significant negative relationships were observed between BMI and mindfulness $(p=.050)$, mindful eating and self-compassion $(p<.001)$. Motivations to eat palatable foods displayed a significant negative relationship to mindfulness mindful eating and self-compassion.

Inter-correlations between mindfulness, mindful eating, self-compassion, and motives to eat palatable foods subscales, as well as BMI, are presented in Table 2. BMI displayed a significant negative relationship with the acceptance, non-reactivity, distractibility and unstructured eating subscales of the mindful eating questionnaire, as well as the acting with awareness and describing subscales of the mindfulness scale. These subscales (apart from describing) appear to negatively associate with all motives to eat palatable foods. However, the BMI related positively only to the coping and conformity subscales of the motives to eat palatable foods scale. Apart from the awareness, routine, and observing subscales, the rest inversely relate to the coping subscale of the motives to eat palatable foods.

Inter-correlations between self-compassion, motives to eat palatable foods subscales, and BMI are presented in Table 3 . The only subscale that relates to BMI was mindfulness. Interestingly, enhancement appears to relate to the negative aspects of self-compassion (i.e., self-judgment, isolation, and over-identification), while coping related to all subscales. Over-identification (i.e., the opposite of mindfulness) was the only self-compassionate subscale that related to all motives to eat palatable foods. Note that multiple regressions were not performed due to collinearity between the predictor variables.

\section{DISCUSSION}

To our knowledge, this is the first investigation to explore mindful eating, mindfulness and self-compassion with motives to eat palatable foods. The aim of this research was two-fold: (a) to investigate the association between mindful eating, mindfulness and

Table 1

Means, standard deviations, and bivariate correlations between mindfulness, mindful eating, self-compassion, motives to eat palatable foods and BMI

\begin{tabular}{|c|c|c|c|c|c|c|}
\hline & 1 & 2 & 3 & 4 & $M$ & $S D$ \\
\hline (1) BMI & & & & & 24.75 & 5.36 \\
\hline (2) PEMS & $.16^{*}$ & & & & 55.09 & 16.06 \\
\hline (3) MES & $-.41^{* *}$ & $-.47^{* *}$ & & & 75.94 & 12.21 \\
\hline (4) SCS & $-.19^{*}$ & $-.29^{* *}$ & $.42^{* *}$ & & 82.68 & 10.15 \\
\hline (5) FFMQ & $-.18^{*}$ & $-.29^{* *}$ & $.40^{* *}$ & $.56^{* *}$ & 73.13 & 12.79 \\
\hline
\end{tabular}

Note. PEMS - Palatable Eating Motives Scale; BMI - body mass index; SCS - Self-Compassion Scale; FFMQ - Five-Facet Mindfulness Questionnaire; MES - Mindful Eating Scale.

${ }^{* *} p<.01 .{ }^{*} p<.05$. 


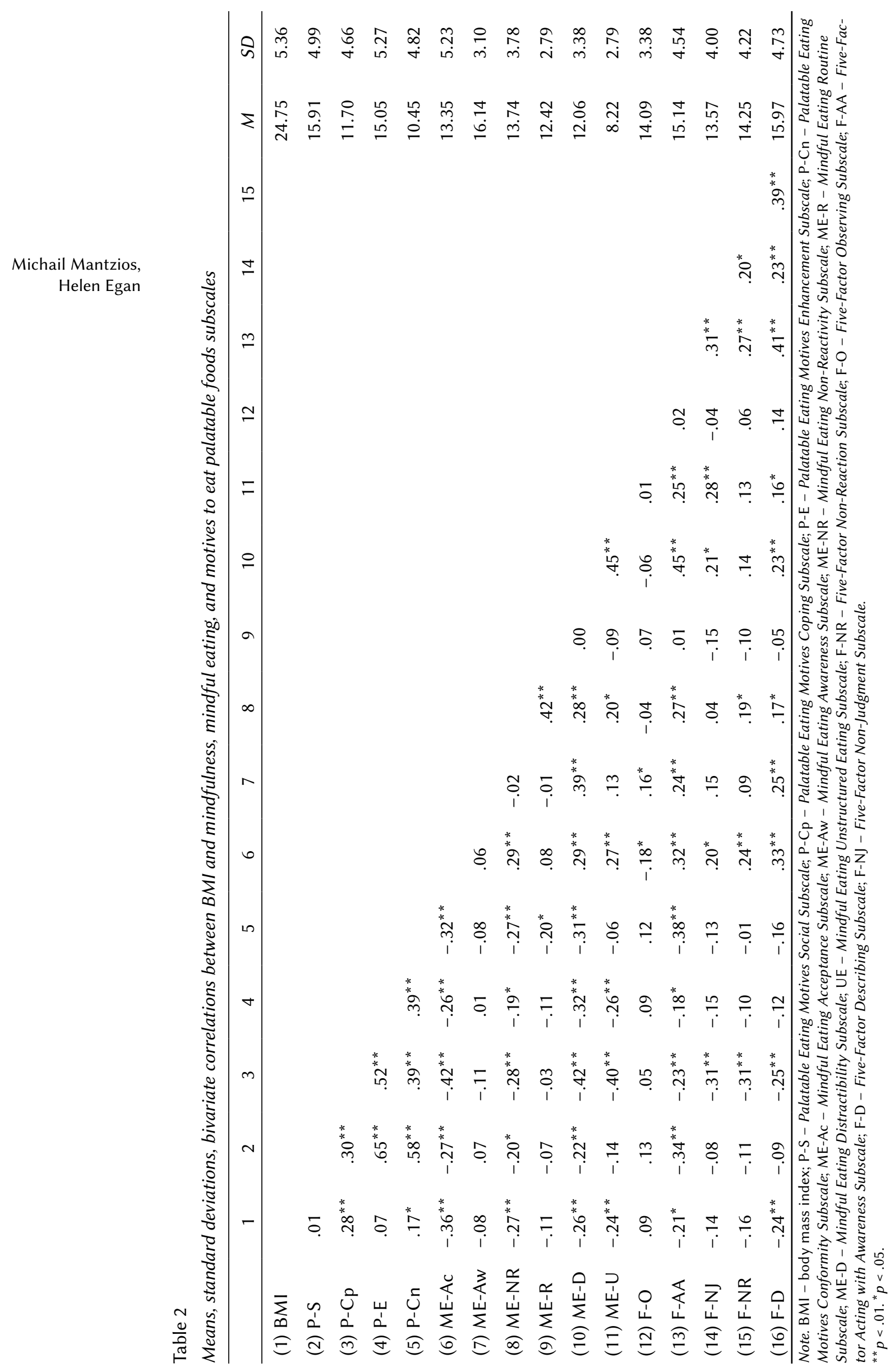


Means, standard deviations, bivariate correlations between BMI, self-compassion, and motives to eat palatable foods subscales

\begin{tabular}{|c|c|c|c|c|c|c|c|c|c|c|c|c|}
\hline & 1 & 2 & 3 & 4 & 5 & 6 & 7 & 8 & 9 & 10 & $M$ & $S D$ \\
\hline (1) BMI & & & & & & & & & & & 24.75 & 5.36 \\
\hline (2) P-S & .01 & & & & & & & & & & 15.91 & 4.99 \\
\hline (3) P-Cp & $.28^{* *}$ & $.30^{* *}$ & & & & & & & & & 11.70 & 4.66 \\
\hline (4) P-E & .07 & $.65^{* *}$ & $.52^{* *}$ & & & & & & & & 15.05 & 5.27 \\
\hline (5) P-Cn & $.17^{*}$ & $.58^{* *}$ & $.39^{* *}$ & $.39^{* *}$ & & & & & & & 10.45 & 4.82 \\
\hline (6) SK & -.15 & .04 & $-.27^{* *}$ & -.03 & -.05 & & & & & & 14.02 & 5.04 \\
\hline (7) $\mathrm{SJ}$ & .14 & .14 & $.46^{* *}$ & $.26^{* *}$ & $.19^{*}$ & $-.65^{* *}$ & & & & & 12.96 & 4.91 \\
\hline (8) $\mathrm{CH}$ & -.16 & -.04 & $-.30^{* *}$ & -.14 & -.06 & $.70^{* *}$ & $-.49^{* *}$ & & & & 12.34 & 4.36 \\
\hline (9) I & .11 & $.20^{*}$ & $.40^{* *}$ & $.27^{* *}$ & .14 & $-.51^{* *}$ & $.72^{* *}$ & $-.49^{* *}$ & & & 10.53 & 4.07 \\
\hline (10) M & $-.20^{*}$ & -.05 & $-.34^{* *}$ & -.11 & -.08 & $.76^{* *}$ & $-.55^{* *}$ & $.77^{* *}$ & $-.56^{* *}$ & & 12.50 & 3.85 \\
\hline (11) $\mathrm{OI}$ & .14 & $.22^{* *}$ & $.52^{* *}$ & $.36^{* *}$ & $.23^{* *}$ & $-.53^{* *}$ & $.73^{* *}$ & $-.50^{* *}$ & $.76^{* *}$ & $-.62^{* *}$ & 10.14 & 3.93 \\
\hline
\end{tabular}

Motivations to eat palatable foods and mindfulness-based constructs

Note. BMI - body mass index; P-S - Palatable Eating Motives Social Subscale; P-Cp - Palatable Eating Motives Coping Subscale; P-E - Palatable Eating Motives Enhancement Subscale; P-Cn - Palatable Eating Motives Conformity Subscale; SK - Self-Kindness Subscale; SJ - Self-Judgment Subscale; CH - Common Humanity Subscale; I - Isolation Subscale; M - Mindfulness Subscale; OI Over-Identification Subscale.

${ }^{* *} p<.01 .{ }^{*} p<.05$.

self-compassion with BMI and motives to eat palatable foods, and (b) to investigate BMI and motives to eat palatable foods in relation to the subscales of the mindfulness, mindful eating, and self-compassion scales. The findings initially suggested that there was a significant positive relationship between BMI and motivations to eat palatable foods, which is comparable with previous findings (Boggiano et al., 2014; Burgess et al., 2014). As expected, significant negative relationships were observed between BMI and mindfulness, as well as self-compassion, with an even stronger relationship with mindful eating. Motivations to eat palatable foods displayed a significant negative relationship with mindfulness and mindful eating and self-compassion, again, with mindful eating being the most significant. The results support prior research that identified that mindfulness, self-compassion and mindful eating relate to lower BMI and healthier eating (Camilleri et al., 2015; Taylor, Daiss, \& Krietsch, 2015), and correspond to the suggestions made of more eating related constructs to be used in eating and weight regulation research (see Mantzios \& Wilson, 2015a). The results were further scrutinized by considering the subscales of all scales.

BMI displayed a significant negative relationship with the acceptance, non-reactivity, distractibility and unstructured eating subscales of the mindful eating questionnaire, as well as the acting with awareness and the describe subscales of the mindfulness scale. Apart from the describing subscale, the aforementioned subscales related negatively with all motives to eat palatable foods. However, BMI related positively only to the coping and conformity subscales of the motives to eat palatable foods scale, which is consistent with past research which explored female participants (Boggiano, 2016). Furthermore, apart from awareness, routine, and the observing subscales, the remaining mindfulness subscales inversely related to the coping subscale of the motives to eat palatable foods. In regards to self-compassion, the only subscale that related to BMI was mindfulness. Interestingly, enhancement appeared to relate positively to self-judgment, isolation, and over-identification, while coping related to all subscales. These findings related to self-compassion are in agreement with theories of self-compassion. Enhancement was identified as a motive that positively related to self-judgment, isolation and over-identification and is consistent with the notion of being 'strict with oneself' to achieve a goal, which is fundamentally different to the principles of self-compassion (Neff, 2011). Self-compassion is an element of acceptance and tolerance of suffering, and all self-compassionate subscales should logically relate to the coping subscale of the motivations to eat palatable foods. The enhancement subscale of the motivations to eat palatable foods should relate to the negative aspects more strongly, as enhancement is indeed more aligned to a self-judgmental and self-critical attitude. Over-identification (i.e., the opposite of mindfulness) was the only subscale within self-compassion that related positively to all motives to eat palatable foods. In other words, the lack of mindfulness at times of suffering appears to relate 
Michail Mantzios, Helen Egan to any motives to eat that are not related to internal cues and hunger.

Overall, the findings suggest that mindfulness, self-compassion and mindful eating are beneficial in reducing motivation to eat palatable foods. While motives to eat palatable foods suggest an intriguing and new element for future research, particularly in association with mindfulness practices, they also suggest the potential to tackle motives that influence weight regulation. This is evident not only through the negative associations of all mindfulness, mindful eating and self-compassion scales, but also by the association with the sub-scales.

Future research should explore the implementation of interventions that relate to mindfulness, mindful eating and/or self-compassion practices, and investigate the potential shift in motives. The superiority of the mindful eating scale is consistent with the description of behavior-specific measurements and interventions described in other research (Hilbert et al., 2015; Mantzios \& Wilson, 2015a), but may also suggest that the re-orientation towards hunger and satiety as healthier motives may be a method of minimizing all other motives. Therefore, motivations to eat palatable foods suggest another method of evaluation and progression of mindfulness practices for weight management, and directives for further longitudinal and cross-sectional explorations are required.

\section{LIMITATIONS}

There were several limitations to the present study. First, the analyses were cross-sectional and require further explorations through experimental investigations. Second, the sample consisted of undergraduate students, which is not representative of the population. Follow-up research should firstly explore and replicate the results with community and clinical populations, to enhance our understanding of obesity and weight regulation, and explore how motives may be similar (or different) with clinical and non-clinical populations. This may provide directions for individual and collective mindfulness-based interventions to counter the current and widespread obesity epidemic.

\section{CONCLUSION}

Overall, the findings suggest that the two main motives (i.e., coping and enhancement) that relate positively to greater BMI relate negatively to mindful eating, mindfulness and self-compassion. In the absence of cultures resistant to the obesity epidemic (e.g., $\mathrm{Hu}, 2005)$, past inconsistent findings, and the mixed relationships to mindfulness-based constructs and interventions, these findings suggest an additional interpretation of health and wellbeing in the field of weight regulation and third-wave practices proposed against the obesity epidemic.

\section{ACKNOWLEDGEMENTS}

Special thanks to Helen's children, who are patiently supporting their mom while she is writing and editing manuscripts. Special thanks to the editor and reviewers for their guidance to finalize this manuscript.

Author contributions: MM identified the topic of potential interest and wrote the first draft of the manuscript. HE discussed the topic area before commencing data collection and wrote and edited the manuscript several times with MM before reaching the final version.

\section{REFERENCES}

Alberts, H. J., Mulkens, S., Smeets, M., \& Thewissen, R. (2010). Coping with food cravings. Investigating the potential of a mindfulness-based intervention. Appetite, 55, 160-163.

Baer, R. A., Smith, G. T., Hopkins, J., Krietemeyer, J., \& Toney, L. (2006). Using self-report assessment methods to explore facets of mindfulness. Assessment, 13, 27-45.

Boggiano, M. M. (2016). Palatable Eating Motives Scale in a college population: Distribution of scores and scores associated with greater BMI and binge-eating. Eating Behaviors, 21, 95-98.

Boggiano, M. M., Burgess, E. E., Turan, B., Soleymani, T., Daniel, S., Vinson, L. D., ...Morse, A. (2014). Motives for eating tasty foods associated with binge-eating: Results from a student and a weight loss seeking population. Appetite, 83, 160-166.

Boggiano, M. M., Wenger, L. E., Turan, B., Tatum, M. M., Morgan, P. R., \& Sylvester, M. D. (2015). Eating tasty food to cope: Longitudinal association with BMI. Appetite, 87, 365-379.

Bohlmeijer, E., Klooster, T. P. M., Fledderus, M., Veehof, M. M., \& Baer, R. (2011). Psychometric properties of the Five Facet Mindfulness Questionnaire in depressed adults and development of a short form. Assessment, 18, 308-320.

Burgess, E. E., Turan, B., Lokken, K. L., Morse, A., \& Boggiano, M. M. (2014). Profiling motives behind hedonic eating. Preliminary validation of the Palatable Eating Motives Scale. Appetite, 72, 66-72

Camilleri, G. M., Méjean, C., Bellisle, F., Hercberg, S., \& Péneau, S. (2015). Association between Mindfulness and Weight Status in a General Population from the NutriNet-Santé Study. PloS One, 10, e0127447. doi: 10.1371/journal.pone.0127447

Daubenmier, J., Lin, J., Blackburn, E., Hecht, F. M., Kristeller, J., Maninger, N., Kuwata, M., Bacchet- 
ti, P., Havel, P.J., \& Epel, E. (2012). Changes in stress, eating, and metabolic factors are related to changes in telomerase activity in a randomized mindfulness intervention pilot study. Psychoneuroendocrinology, 37, 917-928.

Ferrières, J. (2004). The French paradox: lessons for other countries. Heart, 90, 107-111.

Fuller, N. R., Sainsbury, A., Caterson, I. D., Enarsson, M., Denyer, G., Manns, C., ...Markovic, T. P. (2016). Examining mindfulness as a predictor of weight loss - Findings from the DIABEGG study. Obesity Research \& Clinical Practice. Online First, 1-9. doi: 10.1016/j.orcp.2016.03.004

Grossman, P., \& Van Dam, N. T. (2011). Mindfulness, by any other name...: trials and tribulations of sati in western psychology and science. Contemporary Buddhism, 12, 219-239.

Hilbert, A., Braehler, E., Schmidt, R., Löwe, B., Häuser, W., \& Zenger, M. (2015). Self-compassion as a resource in the self-stigma process of overweight and obese individuals. Obesity Facts, 8, 293-301.

$\mathrm{Hu}$, F. B. (2005). Overweight and increased cardiovascular mortality no French paradox. Hypertension, 46, 645-646.

Hulbert-Williams, L., Nicholls, W., Joy, J., \& Hulbert-Williams, N. (2014). Initial validation of the mindful eating scale. Mindfulness, 5, 719-729.

Jordan, C. H., Wang, W., Donatoni, L., \& Meier, B. P. (2014). Mindful eating: Trait and state mindfulness predict healthier eating behavior. Personality and Individual Differences, 68, 107-111.

Kabat-Zinn, J. (1990). Full catastrophe living: Using the wisdom of your body and mind to face stress, pain, and illness. New York: Delacourt.

Kabat-Zinn, J. (2006). Coming to our senses: healing ourselves and the world through mindfulness. New York: Hyperion.

Kristeller, J. L., \& Hallett, C. B. (1999). An exploratory study of a meditation-based intervention for binge eating disorder. Journal of Health Psychology, 4, 357-363.

Kristeller, J., Wolever, R. Q., \& Sheets, V. (2014). Mindfulness-based eating awareness training (MBEAT) for binge eating: A randomized clinical trial. Mindfulness, 5, 282-297.

Mantzios, M., \& Giannou, K. (2014). Group vs. single mindfulness meditation: exploring avoidance, impulsivity, and weight management in two separate mindfulness meditation settings. Applied Psychology: Health and Well-Being, 6, 173-191.

Mantzios, M., \& Wilson, J. C. (2014). Making concrete construals mindful: A novel approach of developing mindfulness and self-compassion to assist weight loss, Psychology \& Health, 4, 422-441. doi: $10.1080 / 08870446.2013 .863883$

Mantzios, M., \& Wilson, J. C. (2015a). Mindfulness, Eating Behaviours, and Obesity: A review and reflection on current findings, Current Obesity Reports, 4, 141-146. doi: 10.1007/s13679-014-0131-x
Mantzios, M., \& Wilson, J. C. (2015b). Exploring mindfulness and mindfulness with self-compassion-centered interventions to assist weight loss: theoretical considerations and preliminary results of a randomized pilot study. Mindfulness, 6, 824835.

Mantzios, M., \& Egan, H. H. (2017). On the role of self-compassion and self-kindness in weight regulation and health behaviour change. Frontiers in Psychology, 8, 229. doi: 10.3389/fpsyg.2017.00229

Mantzios, M., Wilson, J. C., Linnell, M., \& Morris, P. (2015). The role of negative cognitions, intolerance of uncertainty, mindfulness, and self-compassion in weight regulation among male army recruits, Mindfulness, 6, 545-552. doi: 10.1007/s12671-0140286-2

Mason, A. E., Epel, E. S., Kristeller, J., Moran, P. J., Dallman, M., Lustig, R. H., Acree, M., Bacchetti, P., Laraia, B.A., Hecht, F.M., \& Daubenmier, J. (2016). Effects of a mindfulness-based intervention on mindful eating, sweets consumption, and fasting glucose levels in obese adults: data from the SHINE randomized controlled trial. Journal of Behavioral Medicine, 39, 201-213.

Neff, K. D. (2003a). The development and validation of a scale to measure self-compassion. Self and Identity, 2, 223-250.

Neff, K. D. (2003b). Self-compassion: An alternative conceptualization of a healthy attitude toward oneself. Self and Identity, 2, 85-101.

Neff, K. D. (2011). Self-compassion, self-esteem, and well-being. Social and Personality Psychology Compass, 5, 1-12. doi: 10.1159/000215071

Schoenefeld, S. J., \& Webb, J. B. (2013). Self-compassion and intuitive eating in college women: Examining the contributions of distress tolerance and body image acceptance and action. Eating Behaviors, 14, 493-496. doi: 10.1016/j.eatbeh.2013.09.001

Taylor, M. B., Daiss, S., \& Krietsch, K. (2015). Associations among self-compassion, mindful eating, eating disorder symptomatology, and body mass index in college students. Translational Issues in Psychological Science, 1, 229-238.
Motivations to eat palatable foods and mindfulness-based constructs 